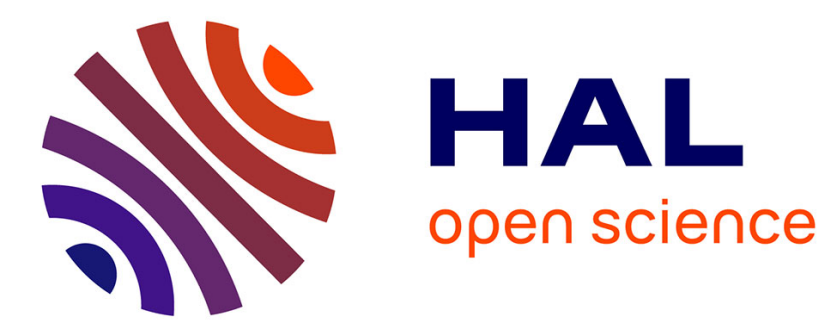

\title{
Sur la mesure des longueurs d'ondulation des radiations infra-rouges
}

L. Mouton

\section{To cite this version:}

L. Mouton. Sur la mesure des longueurs d'ondulation des radiations infra-rouges. J. Phys. Theor. Appl., 1880, 9 (1), pp.113-117. 10.1051/jphystap:018800090011300 . jpa-00237603

\section{HAL Id: jpa-00237603 https://hal.science/jpa-00237603}

Submitted on 1 Jan 1880

HAL is a multi-disciplinary open access archive for the deposit and dissemination of scientific research documents, whether they are published or not. The documents may come from teaching and research institutions in France or abroad, or from public or private research centers.
L'archive ouverte pluridisciplinaire HAL, est destinée au dépôt et à la diffusion de documents scientifiques de niveau recherche, publiés ou non, émanant des établissements d'enseignement et de recherche français ou étrangers, des laboratoires publics ou privés. 


\section{SUR LA MESURE DES LONGUEURS D'ONDULATION DES RADIATIONS INFRA-ROUGES ;}

\section{Par M. L. MOUTON.}

Ainsi que je l'ai rappelé dans un premier article ( 1 ), si entre deux nicols parallèles on place une lame de quartz taillée parallèlement à son axe, celui-ci faisant un angle de $45^{\circ}$ avec les sections principales des nicols, et que l'on fasse traverser le système par un faisceau normal à la lame, reçu ensuite sur la fente d'un spectroscope, le spectre sera sillonné de bandes noires parallèles à la fente, et la longueur d'onde $\lambda$ du milieu de chacune de ces bandes répondra à la relation

$$
\frac{e\left(n^{\prime}-n\right)}{\lambda}=\frac{2 k+1}{2}
$$

dans laquelle $e$ est l'épaisseur de la lame, $n^{\prime}$ et $n$ les indices ordinaire et extraordinaire du quartz correspondant à $\lambda, k$ un nombre entier qui diminue d'une unité quand on passe d'une bande à sa voisine en allant du violet au rouge. J'ai montré comment se détermine l'épaisseur $e$ en millimètres de Fraunhofer $\left({ }^{2}\right)$, ainsi que le nombre $k$ correspondant à une bande déterminée. Ainsi, j’ai cité une lame pour laquelle $e=247^{*}$, et le nombre $\frac{2^{k}+\mathrm{I}}{2}$ correspondant à la dernière bande noire visible du côté rouge est $\frac{7}{2}$. Il suit de là que les bandes obscures ou plutôt froides que MM. Fizeau et Foucault ont les premiers rues se continuer dans le spectre infra-rouge ne pourront pas, avec cette plaque, dépasser trois, correspondant successivement aux nombres $\frac{5}{2}, \frac{3}{2}$ et $\frac{\mathrm{I}}{2}$.

Cela posé, supposons un spectroscope agencé de telle façon qu'on puisse connaître toujours avec une exactitude déterminée l'indice,

(') Journal de Physique, t. VIII, p. 393 ; 1879 .

(2) J'appelle millimètre de Fraunhofer une unité de longueur telle que la longueur d'ondulation de la raie $D_{1}$ y soit o, o005888, nombre adopté par M. Mascart. J'en désigne la millième partie par $\mu$.

J. de Phys., t. IX. (Avril r880.) 
par rapport à la substance du ou des prismes qui y entrent, de la radiation frappant la pile à un moment donné; faisons en second lieu les prismes du spectroscope en quartz, l'arête réfringente étant parallèle à l'axe de cristallisation. Suivant que l'on placera les sections principales des polariseurs parallèles ou perpendiculaires à cette arête, on aura le spectre extraordinaire ou le spectre ordinaire du quartz. Puisque dans l'un comme dans l'autre cas nous pouvons mesurer l'indice des radiations frappant la pile, celle-ci étant amenée sur une bande froide dans le cas du spectre ordinaire, on aura le nombre $n$, et sur la bande de même ordre, dans le cas du spectre extraordinaire, le nombre $n^{\prime}$. Ainsi, sans aucune extrapolation, tout, excepté $\lambda$, sera connu dans l'égalité (I).

Tel est le procédé que j'ai employé; c'est, comme on le voit, celui de M. Fizeau ( 1 ), débarrassé de l'extrapolation relative à la différence $n^{\prime}-n$, dont je mesure directement les deux termes.

Il ne me reste plus qu'à décrire brièvement le spectroscope que j'ai adopté et le moyen à l'aide duquel la pile était amenée sur le milieu d'une bande froide.

La fig. I représente tout le dispositif expérimental.

Fig. 1.

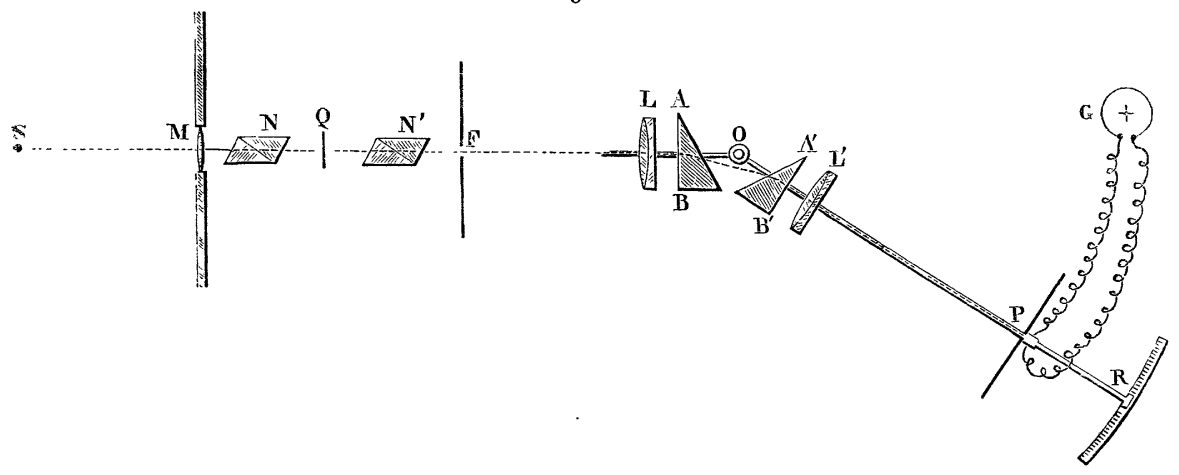

En S est la source lumineuse et calorifique (lampe Bourbouze), dans la pièce voisine de celle qui contient les appareils; une lentille $\mathrm{M}$ encastrée dans la cloison donne en $\mathrm{F}$ l'image de la lampe.

(1) Comptes rendus de la Société philomathique, 1847. 
Entre $\mathrm{M}$ et $\mathrm{F}\left(\mathrm{o}^{\mathrm{m}}, 5\right.$ o environ ) sont les polariseurs $\mathrm{N}$ et $\mathrm{N}^{\prime}$, énormes nicols de $o^{\text {ma }}, 05$ d'ouverture, et la lame Q. En F commence le spectroscope. La fente $\mathrm{F}$ et la lentille achromatique $\mathrm{L}$ constituent le collimateur; la lentille $\mathrm{L}^{\prime}$ et la pile linéaire $\mathrm{P}$ correspondent à la lunette. A et $\mathrm{A}^{\prime}$ sont deux prismes d'angles réfringents égaux : le premier, $\mathrm{A}$, est fixe ; le second, $\mathrm{A}^{\prime}$, estavec la lentille $\mathrm{L}^{\prime}$ et la pile $\mathrm{P}$, monté sur un bras solide tournant autour d'un centre $\mathrm{O}$. En $\mathrm{R}$ est un arc de cercle gradué de centre $\mathrm{O}$, donnant la minute. C'est le dispositif de couple dont l'idée appartient à MM. Gouy et Thol$\operatorname{lon}(1)$.

Je place la fente $\mathbf{F}$ au foyer de la lentille $\mathbf{L}$, en même temps que le faisceau cylindrique qui sort de $\mathrm{L}$ est rendu normal à la face $\mathrm{AB}$ par le procédé le plus exact connu, à savoir l'image de la fente $\mathrm{F}$ réfléchie sur $\mathrm{AB}$ étant ramenée à cö̈ncider avec cette fente ellemême. La même chose a lieu pour $\mathrm{A}^{\prime}, \mathrm{L}^{\prime}$ et $P$. Ici j'ai dù faire en sorte que le corps de la pile pût sc séparer de l'écran-fente donnant accès aux radiations; une lampe mise à la place de la pile fournit lc faisceau, et, le réglage fini, la pile est replacée. Enfin un brûleur à sodium, mis à la place de la lampe $\mathrm{S}$, donne en $\mathrm{P}$ la raie très nette de la soude; j'amène, par la rotation du bras OP autour de $\mathrm{O}$, cette raie à tomber sur la fente de la pile, et la position du vernier terminant ce bras est notée sur l'arc R.

Ce système présente les avantages suivants : $1^{\circ}$ toute radiation lumineuse ou obscure frappant la pile jouit, par rapport au système de prismes, des propriétés focales du minimum de déviation $; 2^{\circ}$ on a pu à l'avance, sur un bon goniomètre, déterminer l'angle $\mathrm{A}$ des prismes, ainsi que leur indice $\nu$ par rapport à la lumière de la soude. La déviation $\Delta$ que le système imprime à celte lumière est alors donnée par la relation

$$
\sin \left(A+\frac{\Delta}{2}\right)=\nu \sin A
$$

et enfin la radiation qui frappera la ligne médiane de la pile quand le vernier sera surl'arc $\mathrm{R}$, à une distance $\delta$ du sodium (du côté rouge

(') Comptes rendus des séances de l'Académie des Sciences, t. LXXXIII, p. 269; t. LXXXVI, p. 329 et 595 . 
par exemple), aura un indice $n$ déterminé par la relation

$$
\sin \left(\mathrm{A}+\frac{\Delta}{2}-\frac{\delta}{2}\right)=n \sin \mathrm{A} \text {. }
$$

Les mesures optiques étant faites à $\mathbf{~} \mathbf{o}^{\prime \prime}$ près avec des prismes se tenant entre $30^{\circ}$ et $35^{\circ}$, si le pointé calorifique fournit $\delta$ à $\mathbf{I}^{\prime}$ près, l'indice $n$ sera déterminé avec quatre chiffres décimaux exacts.

J'ai placé successivement en $\mathrm{Q}$ cinq lames de quartz étudiées optiquement au préalable, comme on l'a vu, et choisies de façon que les bandes qu'elles fournissent ne soient ni trop larges ni trop voisines les unes des autres.

J'ai employé, pour déterminer la position de chaque bande froide, le procédé indiqué par MM. Fizeau et Foucault: la lame $Q$ ayant son axe placé parallèlement aux sections principales des polariseurs, on lit la déviation galvanométrique; la lame est ensuite placée à $45^{n}$, les bandes se produisent et l'on a une nouvelle déviation; le rapport entre celle-ci et la précédente oscille, quand on promène la pile dans le spectre, entre o et 1 ; ses minima correspondent aux bandes froides.

Ce procédé, que MM. Fizeau et Foucault avaient imaginé, à cause de l'irrégularité du spectre calorifique du Soleil pour ne pas, comme ils le disent, “ confondre une bande d'interférence avec un minimum normal de l'intensité calorifique s, avait pour moi l'avantage d'annuler l'effet des variations de la lampe. Grâce à un système automatique permettant de faire tourner rapidement la lame de $45^{\circ}$, chaque couple d'observations, absolument indépendant des autres, ne durait que quelques minutes et pouvait être renouvelé autant qu'il le fallait, en fournissant, quel que soit l'état de la lampe, des résultats toujours comparables entre eux.

Je n'ai recherché exactement que la position des bandes froides, les autres m'ayant toujours paru se déterminer moins nettement.

Le Tableau ci-après donne les résultats suivants. 


\begin{tabular}{|c|c|c|c|c|c|}
\hline $\begin{array}{l}\text { Nos d'ordre } \\
\text { des lames. }\end{array}$ & $\begin{array}{c}\text { Leur } \\
\text { épaisseur. }\end{array}$ & $\begin{array}{c}\text { Ordre } \\
\text { de la dernière } \\
\text { bande } \\
\text { lumineuse. }\end{array}$ & $\begin{array}{c}\text { Ordre } \\
\text { des bandes } \\
\text { calorifiques } \\
\text { pointées. }\end{array}$ & $\begin{array}{l}\text { Valeurs } \\
\text { correspondantes } \\
\text { de } n^{\prime}-n .\end{array}$ & $\begin{array}{c}\text { Valeurs } \\
\text { correspondantes } \\
\text { de } \lambda .\end{array}$ \\
\hline & 125 & & $\left\{\frac{3}{2}\right.$ & o, oogoo & 0,73 \\
\hline $1 \ldots$ & 125 & $\frac{-2}{2}$ & 12 & $o, 00870$ & 2,15 \\
\hline $2 \ldots$ & $18 t$ & $\frac{3}{2}$ & $\frac{3}{2}$ & $o, 0089 o$ & I, 07 \\
\hline $3 \ldots$ & 247 & $\frac{7}{2}$ & $\left\{\begin{array}{l}\frac{5}{2} \\
\frac{3}{2}\end{array}\right.$ & $\begin{array}{l}o, 00893 \\
o, 0088 o\end{array}$ & $\begin{array}{l}0,88 \\
\mathrm{I}, 45\end{array}$ \\
\hline $4 \ldots$ & $3 \circ 3,6$ & $\frac{9}{2}$ & $\left\{\begin{array}{l}2 \\
1 \\
2 \\
3 \\
3\end{array}\right.$ & $\begin{array}{l}o, 00886 \\
0,00875\end{array}$ & $\begin{array}{l}\mathrm{I}, \mathrm{0} 8 \\
\mathrm{I}, 77\end{array}$ \\
\hline 5. . . & 6.6 & $\frac{13}{2}$ & $\frac{5}{2}$ & o, 00868 & 2,I' \\
\hline
\end{tabular}

On voit que la lame $n^{\circ} \mathbf{1}$ a donné toutes les bandes qu'on devait en attendre. Il n'en est pas de même des autres. Ainsi, la bande $\frac{1}{2}$ du $n^{\circ} 3$ n'existe pas. Cela tient à ce qu'elle se produirait à une longueur d'onde que ne renferme pas le spectre des radiations traversant le système.

Il résulte du présent travail qu'un nombre pour ainsi dire illimité de bandes de longueur d'onde connue peuvent être produites dans le spectre infra-rouge donnė par un prisme d'une substance quelconque. On peut donc graduer en longueurs d'onde un spectroscope calorifique, quelle qu'en soit d'ailleurs la forme.

On voit, de plus, que, si dans le spectroscope que j'ai adopté on place des prismes de substances différentes, à côté de la longueur d'onde d'une bande s'obtiendra son indice par rapport à cette substance. Je suis donc actuellement à même de suivre jusqu'à la longueur d'onde $2^{\mu}$, I 4 la loi de dispersion d'une substance quelconque. Si l'on observe que la longueur d'onde de la raie $\mathbf{R}$, la dernière déterminée dans l'ultra-violet par M. Mascart, est $0^{\prime \prime}, 3_{1} 77_{5}$, on voit que celle de la dernière bande que j'ai pointée, $2^{\mu}, I_{4}$, est plus de six fois plus grande. On dispose ainsi d'une gamme totale supérieure à deux octaves et une quarte, dans laquelle ne figure guère que pour une octave la totalité des radiations chimiques et lumineuses. 